\title{
New Water Treatment Chemical PFZSS Preparation and Application
}

\author{
Qilin Liu*
}

College of Tea and Food Science, Wuyi University, Wuyishan 354300, Fujian, China

\begin{abstract}
Nowadays global water quality pollution has become extremely serious, and solving water pollution has been extremely urgent, so researches on water pollution solutions have become even urgent. And flocculating method is the most traditional water purification method, research and create new high performance flocculating agent is one of flocculating method inherent problems. The paper mainly describes adding iron, zinc two kinds of metal captions together in polysyllabic acid, well controlling all aspects requirements in technology, researching and creating new water purification substance - poly iron and zinc silicate sulfate flocculants (PFZSS). PFZSS making method is weighting proper amount sodium silicate into container, and meanwhile putting it into water to dissolve, then utilizing dilute euphoric acid and sodium hydroxide to adjust silicic acid solution $\mathrm{pH}$ value and arrive at regulated scope, letting it to activate and arrive at certain time, slowly importing activated proper silica acid solution into moderate iron sulfate and zinc sulfate on the condition of fully blending, and finally putting and curing for a while. And implement experiment research on how mixed solution metal cations matching; making method, $\mathrm{pH}$ value, and silicon dioxide concentration these factors affect PFZSS overall performance. PFZSS application mainly takes catering industry sewage solution as an example to get PFZSS has very strong absorption capacity, owns good cleaning effects on extremely turbid catering industry treatment sewage COD and turbidity acquiring, its reaction formed flock density is great, sedimentation rate is very fast, so PFZSS will have great application prospects in the aspect of treating urban sewage.
\end{abstract}

Keywords: PFZSS, technological application, water purification, sewage solution.

\section{INTRODUCTION}

Water is an indispensable important part in human and global all species rely to sustain, is irreplaceable extremely precious material resources in industry and agriculture life, production, economic progressive development and environmental improvement and others all living substance cycling process. However, nowadays water pollution has destroyed original substance constituents, since harmful substance mingles with further contaminant that participates in human living substance cycling process, it is a big harm that not only destroys original ecological balance but also brings into great harm to people health and human production and life [1-2].

And on the other hand, Chinese water resources total reserve is nearly 3000 billion cubic meters, which is $6 \%$ of global total water resources, and China water consumption is also one of the most in the world, according to relative statistics, whole nation achieved fresh water in 1993 was nearly 500 billion cubic meters, which was $12 \%$ of global whole year total water intake, comparing to America nationwide fresh water intake as 470 billion cubic meters in 1995, it was much higher [3].

In addition, China has a large population, current Chinese average water resources occupancy volume is less than 3000 cubic meters, roughly is $1 / 4$ of global average per capita water resources occupancy volume, China's per capita average water resources occupancy volume ranks behind the 100th in the world, so it is included in global one of the few per capita average water resources deficiency countries. Besides, China affiliates monsoon climate zones, water resources are uneven distributed in whole nation, south and north natural conditions differences are great; especially current city population rapid increases, natural environment constantly recesses, industry and agriculture water using techniques quite not advanced, waster phenomena is extremely serious, water resources are constantly polluted, which lets original deficient water resources to be worse, and causes hinders to appear in nation economic construction development [4-8].

Therefore water resources reasonable protection, proper application and water quality treatment approach researches have become more and more important.

Nowadays water quality treatment techniques have been more extensive, from which approach of using flocculating agent to precipitate water impurity is a kind of old and simple and economic approach. Flocculent precipitation approach can get rid of water lots of colloidal materials and suspended particles, and play sterilizing roles in water, furthermore, some flocculating agents even play dephosphorization role. Therefore, flocculating agent turns to be more important for water resources purification research [5-8].

Substance in water or other mobile liquid, as long as it can be used to gather and generate flocculent, it can be called as flocculating agent. Flocculating agent can be divided into organic flocculating agent and inorganic flocculating agent 
the two types according to its own chemical constituents. And organic flocculating agent also contains organic polycoagulant and organic polymer flocculent. Inorganic flocculating agent is the most time-honored flocculating agent, though all applied varieties are fewer, its non-toxic, cheap and rich raw materials features can let it to still take no small position in coagulation technique.

Inorganic flocculating agent contains iron sulfate, aluminum sulfate, ferric chloride and so on, from which aluminum sulfate was developed and produced by America in the beginning, and used as an extremely important inorganic flocculating agent up to now. But as industrial demands improve, aluminum sulfate has gradually been replaced by inorganic polymer flocculent. Inorganic polymer flocculent can provide lots of dissociative particles, and possesses very strong absorption ability on colloid particles, and forms colloidal cohesion effects through a series of reaction. And simultaneously generate a certain chemical and physical changes, let colloidal particle and suspended substances surface electric charge to be neutralized, so that let liquid internal contaminant to mutually get closer and adhere to become bigger and form colloidal, further precipitate, at the same time due to sediment superficial area is very large, it possess very strong absorption function, which is an important reason that it is better than other inorganic flocculating agents' effects.

Organic flocculating agent characteristic is very economic, easier to use; but its usage amount is very big, flocculent efficiency is very small, and has drawbacks as very high manufacturing cost, excessive strong corrosivity and else. Organic polymer composed flocculating agent hasn't been developed and widely used until the beginning of the 70s last century, which is a kind of brand new sewage treatment chemical. Comparing with previous flocculating agent, it can double improve application efficiency, and cost is very low, so it tends to gradually be the main using chemical. In addition, its types products quality is stable, organic polymerization flocculating agent output has already occupied sixty percentage of total flocculating agent output. Carry out graft copolymerization on cellulose, starch, chitosan and other natural macromolecules with acrylamide, the polymer achieves good flocculation effects, and meanwhile it possess some particular functions. China developed some chemicals, most are used in sewage treatment and sludge dewatering. Due to compound used acrylamide itself has big toxicity, which can let people nervous centralis to be narcotized, its usage scope has certain restriction so that flocculating agent ought to continue to develop towards cheap, non-toxic and harmless aspect.

To sum up, the paper according to above advantages and disadvantages, takes PFZSS inorganic flocculating agent as research objects, applies reasonable approach, designs descriptive developing process and briefly discusses its coagulation principle.

\section{PFZSS INORGANIC FLOCCULATING AGENT PREPARATION}

Main reagents can refer to Table $\mathbf{1 .}$

Main experiment apparatus is $\mathrm{Ph}$ meter that is used to test $\mathrm{pH}$ value, photometer that is used to analyze concentration, photoelectricity analytic scale that is used to weight, oven that is used to dry, constant temperature magnetic stirrers and variable speed blender that are used to blend, digital display turbidity meter that is used to make data analysis, thermoregulation multi-purpose furnace and electric blower that are used to heat, and infrared oil analyzer that is used to analyze oil.

Weigh moderate amount sodium silicate and put it into container, and meanwhile put it into water to dissolve, then utilize dilute sulphuric acid and sodium hydroxide to reasonable adjust silicic acid solution $\mathrm{pH}$ value to arrive at regulated scope, activate it and arrive at certain time, slowly import activated moderate polysilicic acid solution into moderate iron sulfate and zinc sulfate accompanying with fully blending, lay out and cure for a while.

During preparation whole order, so as to achieve good result, it must let poly silicic acid polymerization degree to arrive at reasonable polymerization degree scope to the maximum extent so as to ensure acquired special polysilicic acid of great molecular weight and lots of hydroxides amount in surface, and let polysilicic acid and zinc iron ions blending to be proper. Here, it focuses on analyzing how metal cations matching, making method, $\mathrm{pH}$ value, and silicon dioxide concentration affect PFZSS performance.

On the condition that room temperature is moderate, silicon dioxide concentration setting successively is $2 \%$ and $4 \%$, use polysilicic acid solution conversed into jelly state required time to show polymerization reaction rate to analyze $\mathrm{pH}$ value impacts on polymerization whole process. Experiment result refers to Fig. (1).

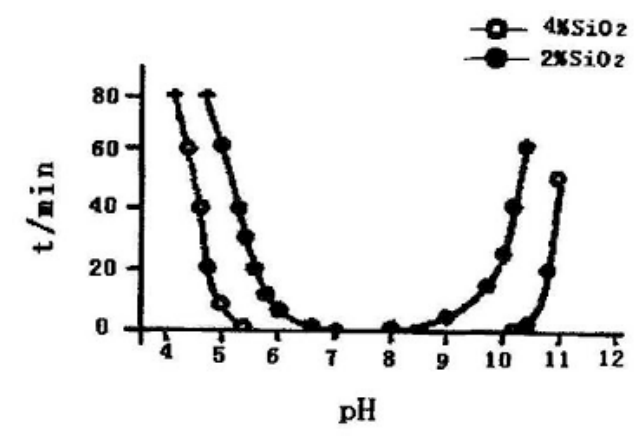

Fig. (1). PH value impacts on polymerization whole process.

By figure, it is clear that in case other setting conditions are the same, $\mathrm{pH}$ value fluctuates surrounding 7 , silicic acid polymerization phenomena occurrence rate is the fastest, while in case $\mathrm{pH}$ value is less than 7 , silicic acid polymerization phenomena occurrence rate but reduces as polymerization occurred previous $\mathrm{pH}$ value gets small, and further indicates that acidity concentration gets stronger, silicic acid polymerization phenomena occurrence rate will become slower. To sum up, it is clear that in the whole process, it can control silicic acid polymerization reaction rate through changing $\mathrm{pH}$ values. The attribute is crucial to the whole process, during production order at ordinary times, when silicic acid polymerization degree arrives at reasonable polymerization degree, blend certain acid or alkali into 
Table 1. Experiment mainly applied reagents

\begin{tabular}{|c|c|c|c|}
\hline Name of reagent & Molecular weight & Content & Pse \\
\hline \hline Sodium silicate & 284.2 & $99.80 \%$ & Preparation \\
\hline Iron sulfate & 399.87 & $99.80 \%$ & Analysis \\
\hline Methylene blue & 373.9 & $98.50 \%$ & Analysis \\
\hline Carbon tetrachloride & 153.82 & $99.80 \%$ & Analysis \\
\hline Potassium dichromate & 294.18 & $99.80 \%$ & Preparation \\
\hline Aluminum sulfate & 666.41 & $99 \%$ & Analysis \\
\hline Sulfuric acid & 98.08 & $95-98 \%$ & Analysis \\
\hline Sodium hydroxide & 40 & $96 \%$ & Preparation \\
\hline Zinc sulfate & 287.56 & Qualified & \\
\hline
\end{tabular}

Table 2. PFZSS preparation experiment table.

\begin{tabular}{|c|c|c|c|c|c|}
\hline Test No. & $\begin{array}{c}\text { Iron and zinc mole } \\
\text { ratio }\end{array}$ & $\begin{array}{l}\text { Iron and silicon } \\
\text { dioxide mole ratio }\end{array}$ & $\begin{array}{l}\text { Dosage } \\
(\mathrm{mg} / \mathrm{L})\end{array}$ & Preparation way & $\begin{array}{l}\text { Turbidity remov- } \\
\text { al rate }\end{array}$ \\
\hline 1 & $0.5(1)$ & $0.5(1)$ & $50(1)$ & Add together (1) & 89.16 \\
\hline 2 & $0.5(1)$ & $1.0(2)$ & $100(2)$ & First add iron and then add zinc (2) & 97.14 \\
\hline 3 & $0.5(1)$ & $1.5(3)$ & $200(3)$ & First add zinc and then add iron (3) & 96.93 \\
\hline 5 & $1.0(2)$ & $1.0(2)$ & $200(3)$ & Add together (1) & 96.93 \\
\hline 6 & $1.0(2)$ & $1.5(3)$ & $50(1)$ & First add iron and then add zinc (2) & 96.73 \\
\hline 7 & $1.5(3)$ & $0.5(1)$ & $200(3)$ & First add iron and then add zinc (2) & 96.32 \\
\hline 8 & $1.5(3)$ & $1.0(2)$ & $50(1)$ & First add zinc and then add iron (3) & 97.96 \\
\hline 11 & 292.84 & 292.44 & 292.03 & 290.80 & \\
\hline 12 & 291.01 & 291.82 & 292.64 & 290.18 & \\
\hline 13 & 94.41 & 94.27 & 94.14 & 95.37 & \\
\hline 14 & 97.61 & 97.48 & 97.34 & 96.93 & \\
\hline 15 & 97 & 97.27 & 97.55 & 96.73 & \\
\hline 16 & 3.2 & 3.21 & 3.41 & 1.57 & \\
\hline
\end{tabular}

polysilicic acid solution that can rapid reduce or rise $\mathrm{pH}$ values so that can reasonable extend storage time.

Iron and zinc mole ratio, iron and silicon dioxide mole ratio, preparation approach and chemical dosing measurement are important indicators in controlling PFZSS performance.

On the condition that room temperature, silicon dioxide mass concentration, $\mathrm{pH}$ value is appropriate, carry out test, observe and get its result, get PFZSS optimum metal ion ratio and its preparation way.
According to above experiment obtained data and result, finally analyze and get that during different preparation processes, PFZSS preparation most reasonable metal collocation proportion is No. 4 test, which is zinc and iron mole ratio being $1: 2$, iron and silicon dioxide mole ratio being $1: 1$, preparation method is dosing together, dosage is $100 \mathrm{mg} / \mathrm{L}$. Dosage, iron and silicon dioxide mole ratio, iron and zinc mole ratio, and preparation approach respectively affect experiment effects from serious to light. 
Table 3. Test of PFZSS handling with restaurant waste water.

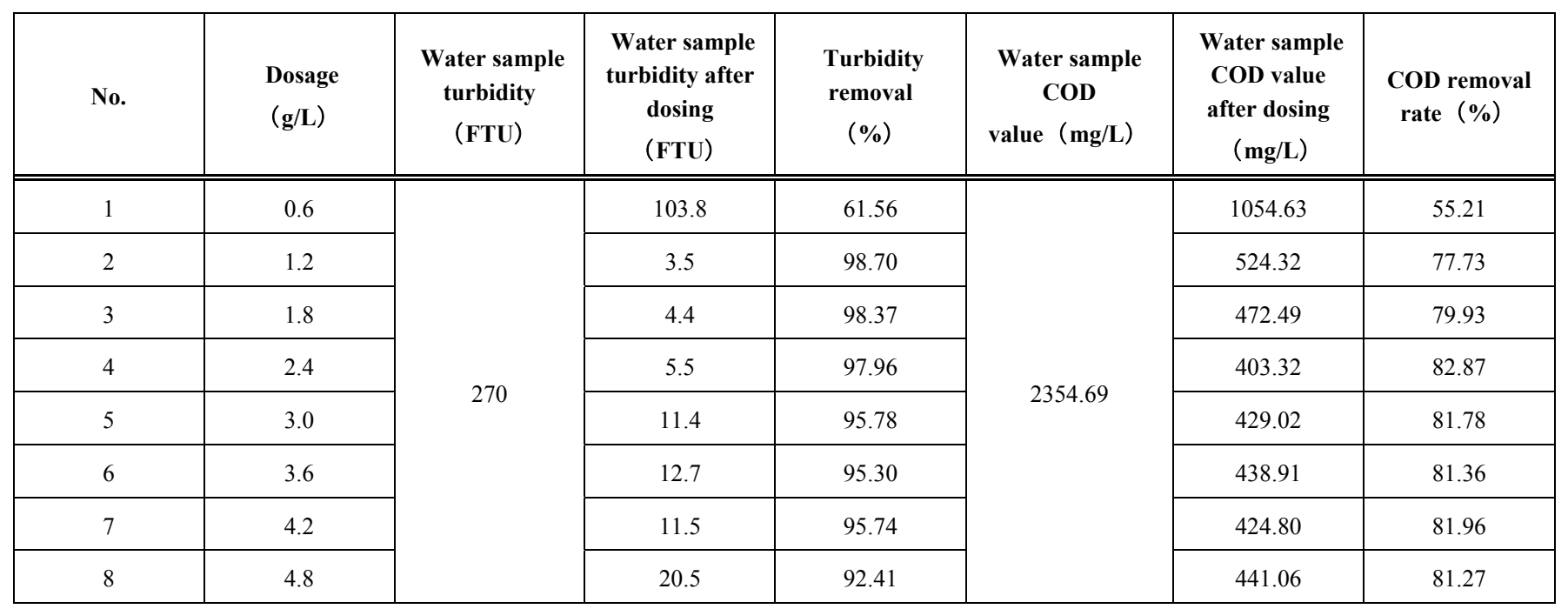

\section{PFZSS INORGANIC FLOCCULATING AGENT APPLICATION}

In order to research on PFZSS cleaning effects in application life, the paper use PFZSS flocculating agent to solve some restaurant waste water, experiment result after removing COD and turbidity is as Table 2.

From experiment, it observes that PFZSS flocculation rate becomes faster after dosing. Experiment shows that turbidity removal effect is the best when PFZSS dosage is $1.8 \mathrm{~g} / \mathrm{L}$, turbidity removal rate is as high as $98 \%$. COD removal rate is best when PFZSS dosage is $2.4 \mathrm{~g} / \mathrm{L}$, the removal rate arrives at $82.87 \%$.

Therefore, it can get following conclusions:

PFZSS has very strong absorption capacity, owns good cleaning affects on extremely turbid catering industry treatment sewage COD and turbidity acquiring, its reaction formed floc density is great, sedimentation rate is very fast, and so PFZSS will have great application prospects in the aspect of treating urban sewage. Table 3 .

\section{CONCLUSION}

During PFZSS preparation whole process, the optimum conditions for preparation are:

1) Silicon dioxide concentration: $3 \%$

2) Solution $\mathrm{pH}$ value: 5

3) Iron and zinc mole ratio: 1:2

4) Iron and silicon dioxide mole ratio : 1:1

5) Preparation way: Dose together
6) All indicators that affect PFZSS performance from primary to secondary are successively dosage, iron and silicon dioxide mole ratio, iron and zinc mole ratio and preparation approach.

PFZSS has very strong absorption capacity, owns good cleaning effects on extremely turbid catering industry treatment sewage COD and turbidity acquiring, its reaction formed floc density is great, sedimentation rate is very fast, and so PFZSS will have great application prospects in the aspect of treating urban sewage.

\section{CONFLICT OF INTEREST}

The authors confirm that this article content has no conflict of interest.

\section{ACKNOWLEDGEMENTS}

Declared none.

\section{REFERENCES}

[1] X. Wei, "PFZSS flocculating agent synthesis and its performance research," Guangdong University of Technology, 2004.

[2] Y. Jian-Feng, "Polysilicate type coagulant paper waste treatment engineering research," China High Technology Enterprises, vol.22, no. 11, pp. 101-115, 2014.

[3] L. Shu-Yun, "Copper-Zinc-Polysilicate flocculating agent preparation and its paper waster application research ,"Hunan University , vol. 25, no. 11, pp. 101-115, 2006

[4] M. J. Focazio, D. W. Kolpin, and K. K. Bames, "A national reconnaissance for phar-maceuticals and other organic wastewater contaminants inthe United States-II: Untreated drinking water sources, "Science of the Total Environment, vol.11, no. 9,pp. 11-19, 2008.

[6] M. J. Focazio, D. W. Kolpin, "Proposed coal-based chemicals project in inner mongolia, " China Chemical Reporter. 2008.

(C) Qilin Liu; Licensee Bentham Open.

This is an open access article licensed under the terms of the (https://creativecommons.org/licenses/by/4.0/legalcode), which permits unrestricted, noncommercial use, distribution and reproduction in any medium, provided the work is properly cited. 